\title{
Refractory acute suppurative thyroiditis secondary to pyriform sinus fistula
}

\author{
Jee Hyue Seo, MD', \\ Yong Hoon Park, MD, PhD', \\ Sei Won Yang, $M^{2}$, \\ Hyun Young Kim, MD ${ }^{3}$ \\ ${ }^{1}$ Department of Pediatrics, \\ Yeungnam University College of \\ Medicine, Daegu, Departments of \\ ${ }^{2}$ Pediatrics and ${ }^{3}$ Surgery, Seoul \\ National University College of \\ Medicine, Seoul, Korea
}

Acute suppurative thyroiditis is a rare disease because the thyroid gland is remarkably resistant to infection. We present a 2-year-old girl with refractory acute suppurative thyroiditis due to a pyriform sinus fistula (PSF). She complained of fever and painful anterior neck swelling. Her condition did not completely improved by multiple parenteral antibiotics along with incision and drainage. Barium esophagogram to detect PSF demonstrated no specific finding. Computed tomography scan showed air bubble superior to the left thyroid gland which indicated a possible fistula connected to the pyriform sinus. An intraoperative laryngoscopy revealed a 2-mm-sized fistula opening. The fistula was successfully treated by chemocauterization with trichloroacetic acid.

\section{Keywords: Thyroiditis, Suppurative, Pyriform sinus, Fistula, Cautery}

\section{Introduction}

Acute suppurative thyroiditis (AST) is a rare infectious disease particularly in childhood because of the high iodine content, rich blood and lymphatic supply and protective fibrous capsule of the thyroid gland ${ }^{1,2)}$. Accordingly, any thyroid or recurrent neck infection in immunocompetent children should raise a suspicion for the presence of predisposing conditions such as pyriform sinus fistula (PSF) or thyroglossal duct remnant. Diagnosis can be made by high resolution ultrasonogram (US), barium studies, computed tomography (CT) scan and endoscopic examination. Treatment with antibiotics and/or incision and drainage $(I \& D)$ is indicated during acute exacerbations. Permanent cure from PSF can only be attained by complete fistulectomy or chemocauterization.

\section{Case report}

A 2-year-old girl presented with fever for 1 week and painful anterior neck swelling for 4 days. Palpation revealed a $4-\mathrm{cm}-\times 3-\mathrm{cm}$-sized anterior neck mass with tenderness and erythema of the overlying skin. Laboratory tests showed white blood cell count of $20,540 / \mu \mathrm{L}$ with shift to the left. C-reactive protein (CRP) was $17.6 \mathrm{mg} / \mathrm{dL}$ and erythrocyte sedimentation rate (ESR) was $120 \mathrm{~mm} / \mathrm{hr}$. Thyroid-stimulating hormone (TSH) was $1.75 \mathrm{mIU} / \mathrm{L}$ (normal range, 0.7-6.4 mIU/L), total T3 was $1.25 \mathrm{mmol} / \mathrm{L}$ (normal range, $1.54-4 \mathrm{mmol} / \mathrm{L}$ ) and free-T4 was $20.32 \mathrm{pmol} / \mathrm{L}$ (normal range, 10-28 pmol/L). Thyroid scan showed diffusely decreased uptake of Tc-99m pertechnetate in the thyroid gland (Fig. 1). US demonstrated an enlarged thyroid gland with diffuse heterogeneous parenchymal echotexture and multiple reactive lymph node enlargement (Fig. 2). The fever subsided one day after intravenous cefotetan treatment, but the neck swelling persisted. Recurrence of the fever and fluctuation of the overlying skin on the swollen neck were noted on the 7th day of hospitalization. CT scan revealed multiple abscesses in both lobes of the thyroid gland without definite evidence of fistula connected to the pyriform sinus (Fig. 3). Antibiotics were changed to meropenem, teicoplanin and clindamycin. 
US-guided fine needle aspiration and I\&D were performed on the 9th and 18th day, respectively. On 20th day, follow-up thyroid function test showed hypothyroidism. (TSH was 48.23 $\mathrm{mIU} / \mathrm{L}$, total T3 was $1.51 \mathrm{mmol} / \mathrm{L}$ and free-T4 was $5.06 \mathrm{pmol} /$ L). Levothyroxine was prescribed at a dose of $3 \mathrm{mcg} / \mathrm{kg}$. Pus continued to drain through the incision site even though the fever subsided. On the 32th day, removal of granulation tissues at the I\&D site and drainage of pus was done. No bacteria was identified on three consecutive pus cultures. On the 37 th day, she was trasferred to Seoul National University Hospital for further evaluation of the underlying deformity. Two esophagograms were unable to demonstrate fistula or other anatomical defect (Figs. 4, 5). Follow-up CT scan was taken because of continuous drainage of pus with erythema and tenderness of the skin. Air bubble superior to the left thyroid gland was discovered, suggestive of a fistula connected to the pyriform sinus (Fig. 6). Two-mm-sized fistula was identified and was successfully

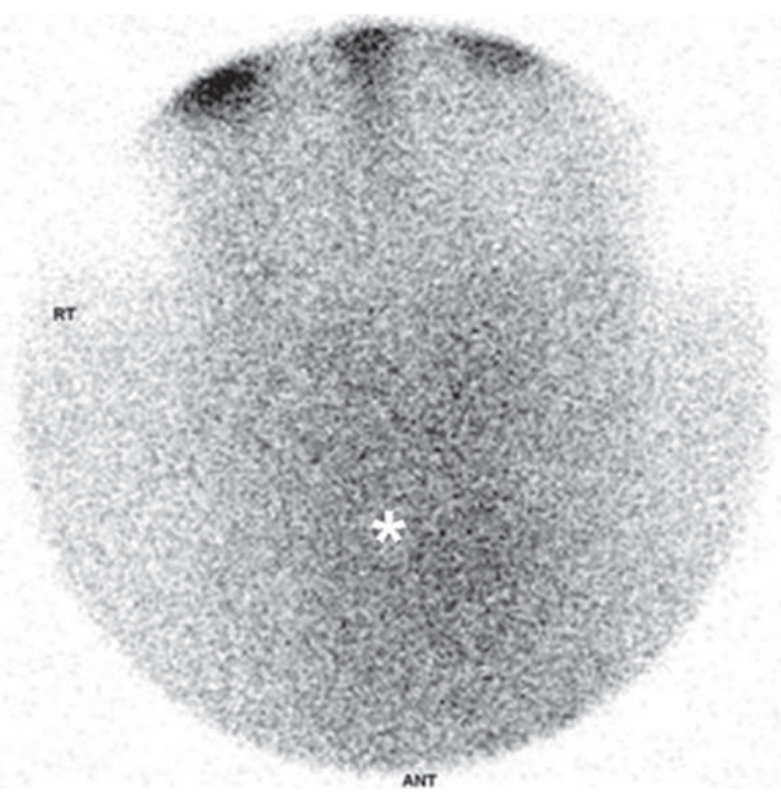

Fig. 1. Thyroid scan shows decreased uptake of Tc-99m pertechnetate in the diffuse area of the thyroid gland.

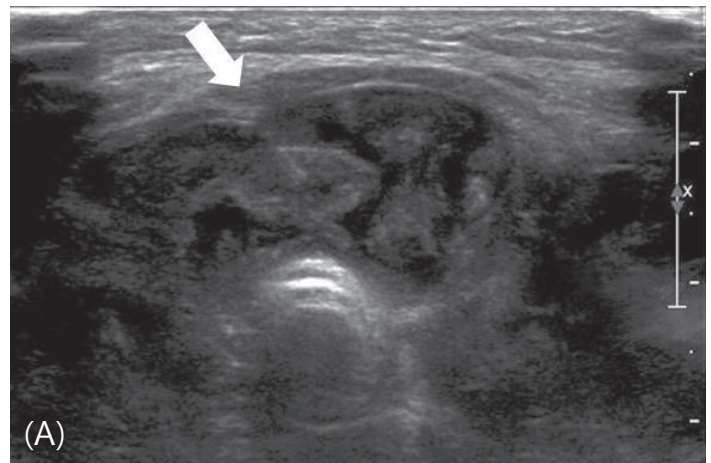

treated with chemocauterization using trichloroacetic acid by intraoperative laryngoscopy. The patient was discharged 5 days after the operation. Levothyroxine was stopped 1 week after the operation, and thyroid function test performed after 1week was normal. TSH was $3.9 \mathrm{mIU} / \mathrm{L}$, total T3 was $78.2 \mathrm{ng} / \mathrm{dL}$ (normal range, $58-159 \mathrm{mmol} / \mathrm{L}$ ) and free-T4 was $0.93 \mathrm{ng} / \mathrm{dL}$ (normal range, $0.8-2.2 \mathrm{ng} / \mathrm{dL}$ ). No recurrence was observed after 2 years of follow-up.

\section{Discussion}

AST is a rare infectious disease particularly in childhood. Usually, patients with AST have predisposing factors including congenital defect such as PSF or thyroglossal duct cyst, preexisting thyroid disease and compromised immune system ${ }^{3)}$. A wide variety of microbial pathogens such as Staphylococcus, Streptococcus, Hemophilus influenza, Klebsiella, Escherichia coli,

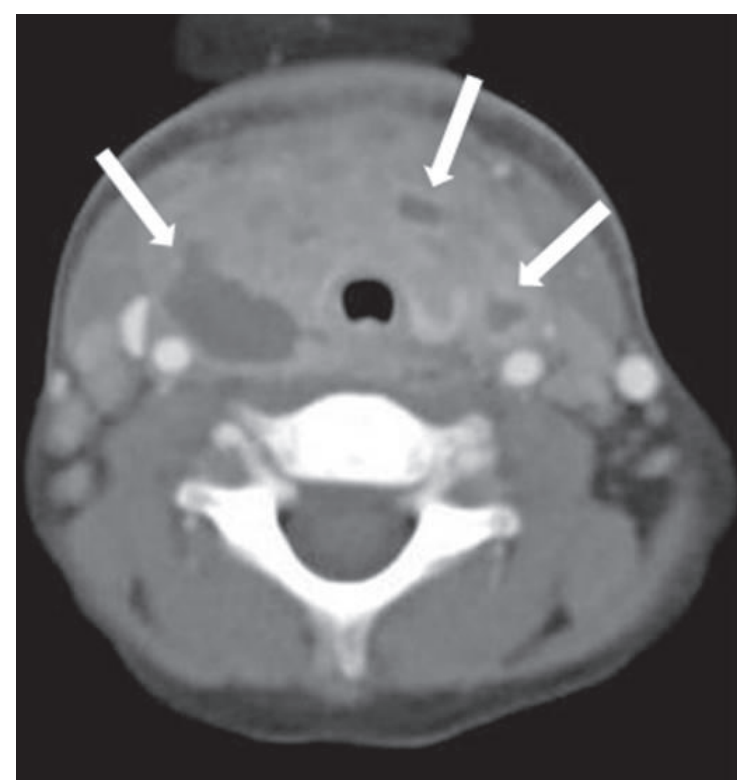

Fig. 3. Computed tomography scan shows multiple hypodense abscesses in both lobes of the thyroid gland.

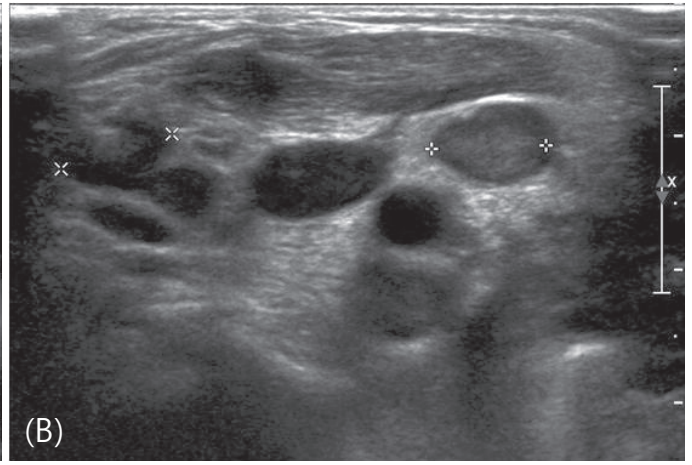

Fig. 2. Ultrasonogram shows (A) enlarged thyroidi gland with diffuse heterogenous echotexture (arrow) and (B) multiple reactive lymph node enlargement. 


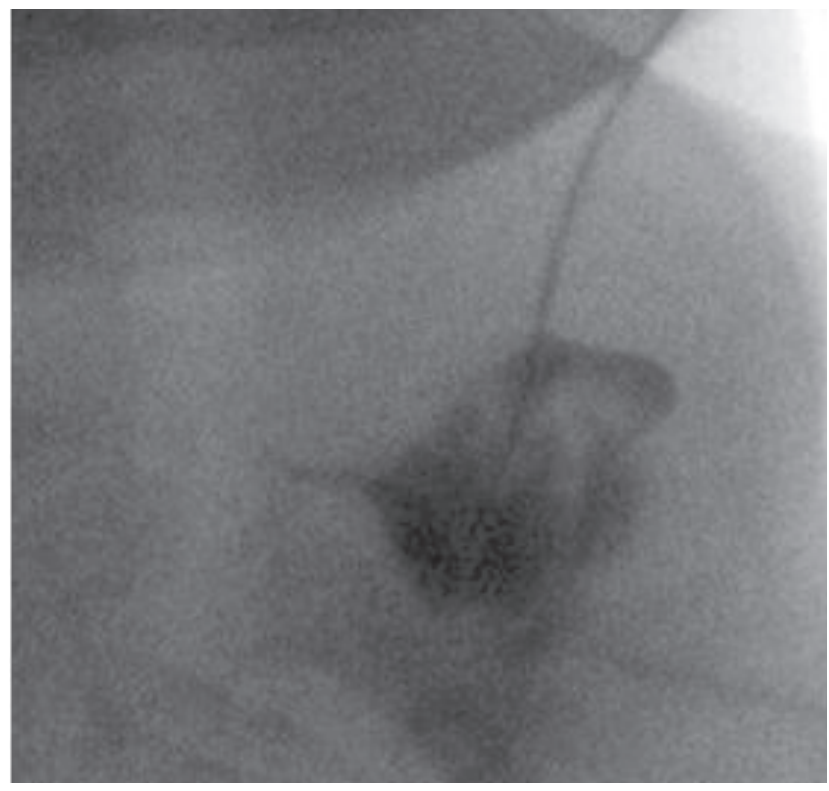

Fig. 4. Contrast was infused through an incision wound at the anterior side of the neck. There was contrast filling at the posterior pharynx, but the pathway was not compatible with a pyriform sinus fistula.

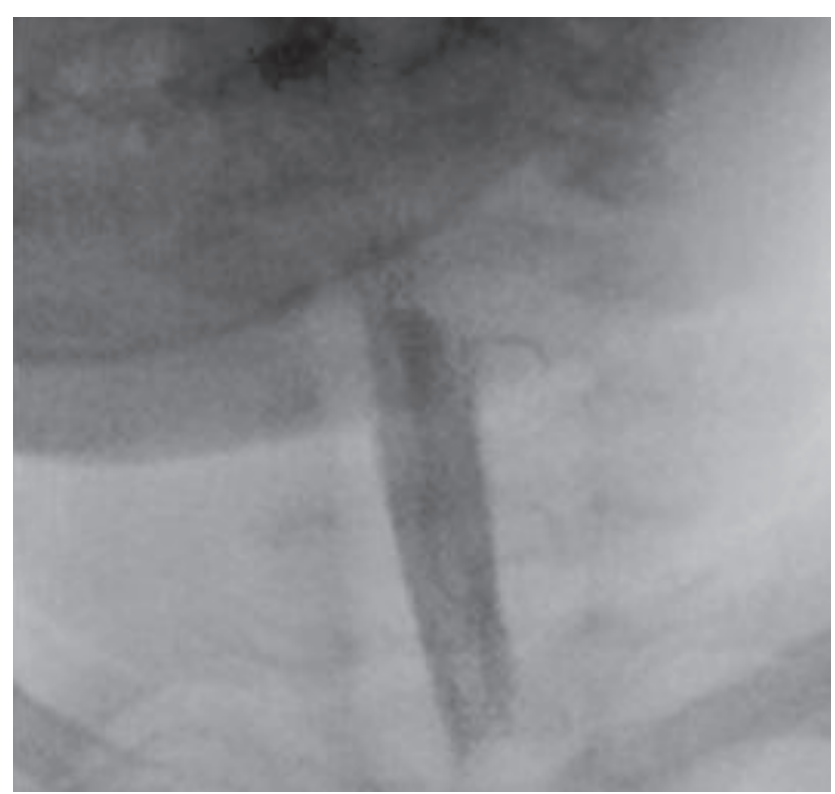

Fig. 5. Esophagogram was checked while swallowing contrast medium. No pyriform sinus fistula tract was demonstrated.

Pseudomonas, Enterobacter, Salmonella and anaerobes which are commonly found as normal flora in the oral cavity, have been reported as causes of AST. Rarely, Mycobacteria, Aspergillus, Coccidioidesimmitis, Cryptococcus neoformans, Histoplasma capsulatum, Candida, Treponema pallidum, and Echinococcus have been identified. As much as $30 \%$ of suppurative thyroiditis are polymicrobial infection ${ }^{1,3,4)}$.

The routes of infection are through blood stream or lymphatics, direct inoculation from adjacent tissue or congenital

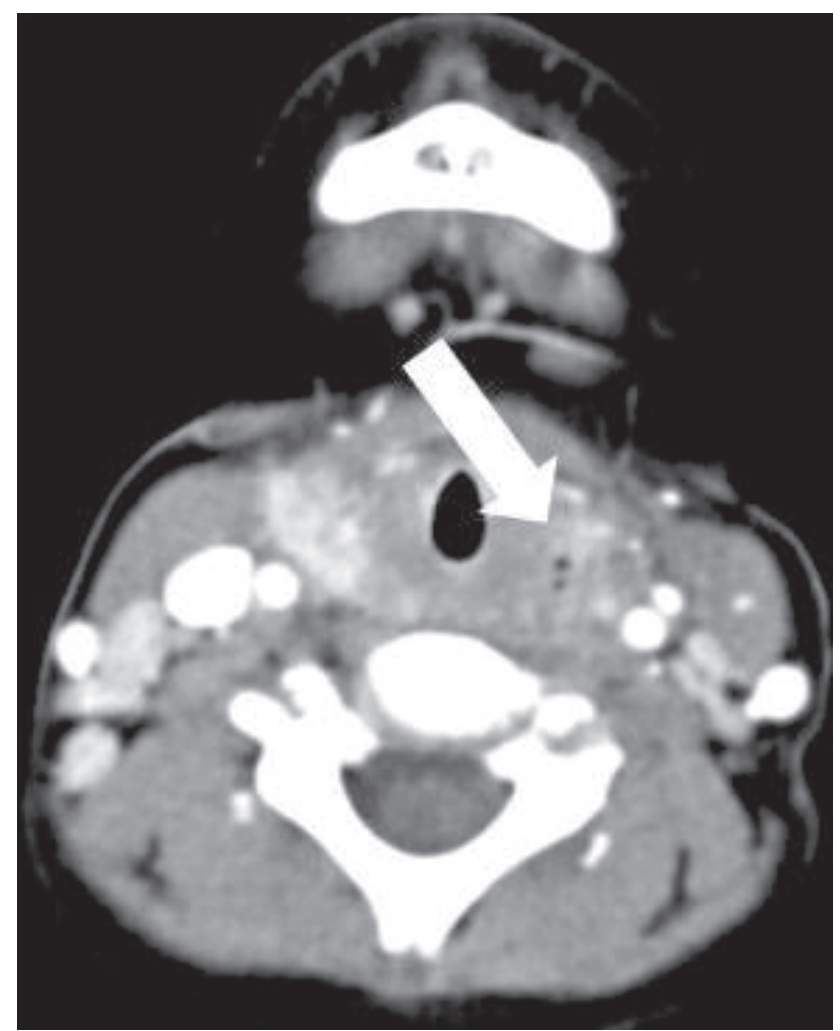

Fig. 6. Neck computed tomography scan shows air bubble superior to the left thyroid gland, which is suspicious for a fistula connected to the pyriform sinus (arrow). The scan also shows slightly decreased extent of the thyroid gland but no interval changes in the bilateral multiple enlarged lymph nodes, suggesting reactive hyperplasia.

anomaly such as PSF which is a remnant of the 3rd or 4th pharyngeal pouch. The sinus tract begins from the apex or base of the pyriform sinus, courses anterio-inferiorly, into either the perithyroid tissue or thyroid parenchyma ${ }^{5,6}$. Because of asymmetric embryological development, it usually located at left side and most often associated with left-sided neck infection. In the acute inflammatory phase, swelling of mucosa and adjacent tissue can cause partial or complete obstruction of the fistula tract and identification the fistula tract may be difficult ${ }^{3}$. Therefore, evaluation for anatomical defect in recurrent thyroiditis or neck infection should be done after management of the acute inflammation ${ }^{2,7)}$. In our case, even though no evidence of PSF was seen at CT scan which was done in acute inflammatory phase, follow up CT scan detected air bubble, suggestive of PSF.

Clinical features of AST include fever, cough, hoarseness, dysphagia, sore throat, anterior neck swelling and limitation of cervical extension ${ }^{1)}$. Both thyroid gland lobes can be affected but the left lobe is more frequently involved. Leukocytosis and increased ESR and CRP are common. Normal thyroid function tests are typical, although transient or permanent hypothyroidism can occur in about $2 \%-3 \%$ of patients and thyrotoxicosis may occur in 5\% as a result of the disease or treatment ${ }^{1,3)}$. US show heterogeneous echogenicity of the thyroid gland with irregular margin and inflammatory changes. CT scan 
is a useful modality for detecting loss of the normal high density of the thyroid gland, abscess formation and air density of a sinus or fistula. Radionulide thyroid scan generally shows absent or decreased uptake in the affected thyroid lobe. An esophagogram can detect underlying anatomical defect. Direct inspection by laryngoscopy or endoscopic hypopharyngoscopy is becoming increasingly useful to identify the internal opening of a PSF and appears to be a more sensitive diagnostic test compared with CT scan and barium esophagogram ${ }^{3,8}$.

Therapy with empiric antibiotics include penicillinase-resistant penicillin or combination of a penicillin and a $\beta$-lactamase inhibitor can improve the AST. Penicillin, clindamycin, combination of macrolide and metronidazole is sometimes recommended. Most suppurative thyroiditis are treated successfully and completely with appropriate antibiotics medication with I\&D although transient alteration of thyroid function may occur. If no clinical improvement occurs after 36-48 hours of therapy, a reassessment is needed to detect the presence of abscess and $\mathrm{PSF}^{1,3)}$. Also, recurrent infection of neck or thyroid gland, especially left sided, evaluation for underlying anomaly such as PSF should be done ${ }^{2,9)}$. Treatment of choice for PSF is elective excision. Recently, electrical or chemical cauterization using silver nitrate, trichloroacetic acid or histoacry have been used in a lot of cases ${ }^{2,3)}$ Most suppurative thyroiditis are treated successfully and completely even though transient altered thyroid function has occurred.

In conclusion, patients with recurrent or refractory AST frequently have an underlying predisposing condition. Esophagogram, CT scan or laryngoscopy should be used to look for an underlying anomaly such as PSF. Besides elective excision, cauterization, which is less invasive, can cure PSF successfully.

\section{Conflict of interest}

No potential conflict of interest relevant to this article was reported.

\section{References}

1. Brook I. Microbiology and management of acute suppurative thyroiditis in children. Int J Pediatr Otorhinolaryngol 2003;67:447-51.

2. Sai Prasad TR, Chong CL, Mani A, Chui CH, Tan CE, Tee WS, et al. Acute suppurative thyroiditis in children secondary to pyriform sinus fistula. Pediatr Surg Int 2007;23:779-83.

3. Paes JE, Burman KD, Cohen J, Franklyn J, McHenry CR, Shoham S, et al. Acute bacterial suppurative thyroiditis: a clinical review and expert opinion. Thyroid 2010;20:24755 .

4. Wongphyat O, Mahachoklertwattana P, Molagool S, Poomthavorn P. Acute suppurative thyroiditis in young children. J Paediatr Child Health 2012;48:E116-8.

5. Hatabu H, Kasagi K, Yamamoto K, Iida Y, Misaki T, Hidaka A, et al. Acute suppurative thyroiditis associated with piriform sinus fistula: sonographic findings. AJR Am J Roentgenol 1990;155:845-7.

6. Nicoucar K, Giger R, Jaecklin T, Pope HG Jr, Dulguerov P. Management of congenital third branchial arch anomalies: a systematic review. Otolaryngol Head Neck Surg 2010;142:21-28.e2.

7. Schneider U, Birnbacher R, Schick S, Ponhold W, Schober E. Recurrent suppurative thyroiditis due to pyriform sinus fistula: a case report. Eur J Pediatr 1995;154:640-2.

8. Fukata S, Miyauchi A, Kuma K, Sugawara M. Acute suppurative thyroiditis caused by an infected piriform sinus fistula with thyrotoxicosis. Thyroid 2002;12:175-8.

9. Yolmo D, Madana J, Kalaiarasi R, Gopalakrishnan S, Kiruba Shankar M, Krishnapriya S. Retrospective case review of pyriform sinus fistulae of third branchial arch origin commonly presenting as acute suppurative thyroiditis in children. J Laryngol Otol 2012;126:737-42. 